\begin{tabular}{|c|c|}
\hline Title & Stability of Ground States in Sectors and Its A pplication to the Wigner-W eisskopf Model \\
\hline Author(s) & A rai, A sao; Hirokawa, Masao \\
\hline Citation & $\begin{array}{l}\text { Reviews in Mathematical Physics, 13(4), 513-528 } \\
\text { https://doi.org/10.1142/S0129055X 01000740 }\end{array}$ \\
\hline Issue Date & 2001-04 \\
\hline Doc URL & http:/hdl.handle.net $/ 2115 / 38266$ \\
\hline Rights & $\begin{array}{l}\text { Electronic version of an article published as Reviews in Mathematical Physics, V ol. 13, Issue 4, 2001, pp. 513-528, } \\
\text { DOI: } 10.1142 \text { S0129055X 01000740 @ W orld Scientific Publishing Company, } \\
\text { http:/www.worldscinet.com/fmp/mp.shtml }\end{array}$ \\
\hline Type & article (author version) \\
\hline File Information & ArHi1.pdf \\
\hline
\end{tabular}

Instructions for use 


\title{
Stability of Ground States in Sectors and Its Application to the Wigner-Weisskopf Model
}

\author{
Asao Arai $^{1}$ and Masao Hirokawa ${ }^{2}$ \\ ${ }^{1}$ Department of Mathematics, Hokkaido University, Sapporo 060-0810, Japan, \\ e-mail: arai@math.sci.hokudai.ac.jp \\ ${ }^{2}$ Department of Mathematics, Faculty of Science, Okayama University, Okayama 700-8530, Japan, \\ e-mail: hirokawa@math.okayama-u.ac.jp
}

\begin{abstract}
We consider two kinds of stability (under a perturbation) of the ground state of a self-adjoint operator, being concerned with (i) the sector to which the ground state belongs and (ii) the uniqueness of the ground state. As an application to the Wigner-Weisskopf model which describes one mode fermion coupled to a quantum scalar field, we prove in the massive case the following: (a) For a value of the coupling constant, the Wigner-Weisskopf model has degenerate ground states ; (b) for a value of the coupling constant, the Wigner-Weisskopf model has a first excited state with energy level below the bottom of the essential spectrum.
\end{abstract}

Mathematics Subject Classifications (2000): 81Q10, 47B25, 47N50

Key Words: Fock space, Wigner-Weisskopf model, ground state, ground state energy, stability, conservation law, first excited state

\section{Introduction}

Let $\mathcal{H}$ be a Hilbert space and $H_{0}$ a self-adjoint operator on $\mathcal{H}$, bounded from below. Let $\mathcal{I}$ be an open interval of $\mathbf{R}$ containing the origin 0 and $\{H(\alpha)\}_{\alpha \in \mathcal{I}}$ be a family of self-adjoint operators acting in $\mathcal{H}$ with $H(\alpha)$ bounded from below for every $\alpha \in \mathcal{I}$ such that

$$
H(0)=H_{0}
$$

For a linear operator $T$ on a Hilbert space, we denote its domain (resp. spectrum, point spectrum) by $D(T)$ (resp. $\sigma(T), \sigma_{\mathrm{p}}(T)$ ). If $T$ is self-adjoint and bounded from below, then

$$
E_{0}(T):=\inf \sigma(T)>-\infty
$$

is called the ground-state energy of $T$. We say that $T$ has a ground state if $\operatorname{ker}\left(T-E_{0}(T)\right) \neq$ $\{0\}$; a non-zero vector in $\operatorname{ker}\left(T-E_{0}(T)\right)$ is called a ground state of $T$. The ground state of $T$ is said to be unique (resp. degenerate) if $\operatorname{dim} \operatorname{ker}\left(T-E_{0}(T)\right)=1$ (resp. $\geq 2$ ). 
In this paper we are concerned with stabilities of ground states of $H(\alpha)$ in the parameter $\alpha \in \mathcal{I}$. In particular we are interested in the following two kinds of stability:

(S.1) (Stability in sectors) Suppose that $\mathcal{H}$ has an orthogonal decomposition

$$
\mathcal{H}=\mathcal{H}_{0} \oplus \mathcal{H}_{1}
$$

with $\mathcal{H}_{j}(j=0,1)$ being a closed subspace of $\mathcal{H}$ such that, for all $\alpha \in \mathcal{I}, H(\alpha)$ is reduced by each $\mathcal{H}_{j}$. In the context of quantum field theory, where $\mathcal{H}$ describes the Hilbert space of state vectors for the model under consideration, each Hilbert space $\mathcal{H}_{j}$ is called a sector. Suppose that $H_{0}$ has a ground state in $\mathcal{H}_{0}$. Then a natural question is: To which sector does the ground states of $H(\alpha)$ belong ?

(S.2) Uniqueness of ground states of $H(\alpha)$.

As for (S.2), there are already fundamental results available (e.g., [Ka, Chapter VII], [RS4, §XII.2]). We apply these results in a more restricted situation to obtain a stronger result.

On the other hand, to our best knowledge, the problem (S.1) seems not to have been considered, at least, on an abstract level.

In Section 2 we prove abstract results on problem (S.1) and degeneracy of ground states. These results are applied to a special class of self-adjoint operators in Section 3. In the last section we consider the Wigner-Weisskopf model (WW model) which describes one mode fermion coupled to a quantum scalar field [WW]. We apply the results of Section 3 to this model in the massive case to establish the following properties: (a) For a value of the coupling constant, the WW model has degenerate ground states ; (b) for a value of the coupling constant, the WW model has a first excited state with energy level below the bottom of the essential spectrum.

\section{Stability of Ground States in Sectors : Abstract Results}

\subsection{Main results}

We denote the resolvent of $H(\alpha)(\alpha \in \mathbf{R})$ by

$$
R_{z}(\alpha):=(H(\alpha)-z)^{-1}, \quad z \in \rho(H(\alpha)),
$$

where $\rho(A)$ denotes the resolvent set of a closed operator $A$. We set

$$
E_{0}(\alpha):=E_{0}(H(\alpha)), \quad \alpha \in \mathcal{I}
$$

Our basic assumptions are as follows: 
(A.1) For all $z \in \mathbf{C} \backslash \mathbf{R}, R_{z}: \alpha \rightarrow R_{z}(\alpha)$ is continuous on $\mathcal{I}$ in operator norm.

(A.2) For each $\alpha \in \mathcal{I}$, there exists a constant $C_{\alpha}>0$ such that, for all sufficiently small $|\kappa|$,

$$
E_{0}(\alpha+\kappa) \geq C_{\alpha}
$$

(A.3) For all $\alpha \in \mathcal{I}, E_{0}(\alpha)$ is an isolated eigenvalue of $H(\alpha)$ (hence $H(\alpha)$ has a ground state).

A solution to the stability problem (S.1) is given in the following theorem:

Theorem 2.1 Assume (A.1)-(A.3) and that $\mathcal{H}$ has the orthogonal decomposition (1.3) such that, for all $\alpha \in \mathcal{I}, H(\alpha)$ is reduced by $\mathcal{H}_{0}$. Suppose that, for all $\alpha \in \mathcal{I}$, the ground state $H(\alpha)$ is unique and that the ground state of $H_{0}$ is in $\mathcal{H}_{0}$. Then, for all $\alpha \in \mathcal{I}$, the ground state of $H(\alpha)$ is in $\mathcal{H}_{0}$.

This theorem can be used to show a degeneracy of ground states:

Corollary 2.2 Assume (A.1)-(A.3) and that $\mathcal{H}$ has the orthogonal decomposition (1.3) such that, for all $\alpha \in \mathcal{I}, H(\alpha)$ is reduced by $\mathcal{H}_{0}$. Suppose that the ground state of $H_{0}$ is unique and in $\mathcal{H}_{0}$. Moreover, suppose that there exists an $\alpha^{\prime} \in \mathcal{I}$ such that $H\left(\alpha^{\prime}\right)$ has a ground state which is not in $\mathcal{H}_{0}$. Then, for some $\alpha_{0} \in \mathcal{I} \backslash\{0\}$, the ground state of $H\left(\alpha_{0}\right)$ is degenerate.

Proof. If the conclusion does not hold, then the ground state $H(\alpha)$ is unique for all $\alpha \in \mathcal{I}$. Hence, by Theorem 2.1, the ground state of $H(\alpha)$ is in $\mathcal{H}_{0}$ for all $\alpha \in \mathcal{I}$. But this contradicts the assumption that $H\left(\alpha^{\prime}\right)$ has a ground state which is not in $\mathcal{H}_{0}$.

To prove Theorem 2.1, we establish two lemmas.

Lemma 2.3 Assume (A.1) and (A.2). Then the ground state energy $E_{0}(\alpha)$ is continuous in $\alpha \in \mathcal{I}$.

Proof. Fix $\alpha \in \mathcal{I}$ arbitrarily. By (A.2), there exists a constant $\gamma_{\alpha} \in \mathbf{R}$ such that, for all sufficiently small $|\kappa|, \gamma_{\alpha} \in \rho(H(\alpha+\kappa))$ and $\gamma_{\alpha}<E_{0}(\alpha+\kappa)$. Assumption (A.1) implies that $\left\|R_{\gamma_{\alpha}}(\alpha+\kappa)-R_{\gamma_{\alpha}}(\alpha)\right\| \rightarrow 0(\kappa \rightarrow 0)$. Hence

$$
\lim _{\kappa \rightarrow 0} \frac{1}{E_{0}(\alpha+\kappa)-\gamma_{\alpha}}=\lim _{\kappa \rightarrow 0}\left\|R_{\gamma_{\alpha}}(\alpha+\kappa)\right\|=\left\|R_{\gamma_{\alpha}}(\alpha)\right\|=\frac{1}{E_{0}(\alpha)-\gamma_{\alpha}},
$$

which implies that $\lim _{\kappa \rightarrow 0} E_{0}(\alpha+\kappa)=E_{0}(\alpha)$. Thus the desired result follows.

Lemma 2.4 Assume (A.1)-(A.3). Suppose that, for all $\alpha \in \mathcal{I}$, the ground state $H(\alpha)$ is unique. Let $\Psi_{0}(\alpha)$ be a normalized ground state of $H(\alpha)$. Then, for all $\alpha \in \mathcal{I}$,

$$
\lim _{\kappa \rightarrow 0}\left(\Psi_{0}(\alpha+\kappa), \Psi_{0}(\alpha)\right) \Psi_{0}(\alpha+\kappa)=\Psi_{0}(\alpha) .
$$


Proof. For each $\alpha \in \mathcal{I}$, we denote by $P_{\alpha}(\cdot)$ the spectral measure of $H(\alpha)$. Fix $\alpha \in \mathcal{I}$ arbitrarily. By (A.3), there exists a constant $a, b \in \mathbf{R} \cap \rho(H(\alpha))$ such that $a<E_{0}(\alpha)<b$ and $(a, b) \cap \sigma(H(\alpha))=\left\{E_{0}(\alpha)\right\}$. By (A.1) and a general fact [RS1, Theorem VIII.23(b)],

$$
\left\|P_{\alpha+\kappa}((a, b))-P_{\alpha}((a, b))\right\| \rightarrow 0(\kappa \rightarrow 0) .
$$

Hence, by [RS4, p.14, Lemma], $\operatorname{dim} \operatorname{Ran} P_{\alpha+\kappa}((a, b))=\operatorname{dim} \operatorname{Ran} P_{\alpha}((a, b))=1$ for all sufficiently small $|\kappa|$. By Lemma 2.3, $E_{0}(\alpha+\kappa) \in(a, b)$ for all $|\kappa|<\delta$ with some constant $\delta>0$. Hence, for all $|\kappa|<\delta, P_{\alpha+\kappa}((a, b))$ is the orthogonal projection onto $\operatorname{ker}(H(\alpha+$ $\left.\kappa)-E_{0}(\alpha+\kappa)\right)$, which implies that $P_{\alpha+\kappa}((a, b)) \Psi_{0}(\alpha)=\left(\Psi_{0}(\alpha+\kappa), \Psi_{0}(\alpha)\right) \Psi_{0}(\alpha+\kappa)$. On the other hand, (2.5) implies that $P_{\alpha+\kappa}((a, b)) \Psi_{0}(\alpha) \rightarrow P_{\alpha}((a, b)) \Psi_{0}(\alpha)=\Psi_{0}(\alpha)(\kappa \rightarrow 0)$. Thus (2.4) follows.

\section{Proof of Theorem 2.1}

Let $\Psi_{0}(\alpha)$ be a normalized ground state of $H(\alpha)$. By the uniqueness of the ground state of $H(\alpha)$, either $\Psi_{0}(\alpha) \in \mathcal{H}_{0}$ or $\Psi_{0}(\alpha) \in \mathcal{H}_{1}$. By the present assumption, $\Psi_{0}(0) \in \mathcal{H}_{0}$.

Suppose that there existed a sequence $\left\{\alpha_{n}\right\}_{n=1}^{\infty}$ such that $\alpha_{n} \rightarrow 0(n \rightarrow \infty)$ and $\Psi_{0}\left(\alpha_{n}\right) \in \mathcal{H}_{1}$. Hence $\left(\Psi_{0}\left(\alpha_{n}\right), \Psi_{0}(0)\right)=0$ for all $n \geq 1$. Then, by applying Lemma 2.4 to the case $\alpha=0$, we have $\Psi_{0}(0)=0$. But this is a contradiction. Thus there exists a constant $\delta>0$ such that, for all $|\alpha|<\delta$, we have $\alpha \in \mathcal{I}$ and $\Psi_{0}(\alpha) \in \mathcal{H}_{0}$.

Let

$$
\alpha_{-}:=\inf \left\{\alpha \in \mathcal{I} \mid \Psi_{0}(\alpha) \in \mathcal{H}_{0}\right\}, \quad \alpha_{+}:=\sup \left\{\alpha \in \mathcal{I} \mid \Psi_{0}(\alpha) \in \mathcal{H}_{0}\right\} .
$$

Then, by the above fact, $\alpha_{-}<0<\alpha_{+}$. We first consider the case $\mathcal{I}=(c, d)$ with $-\infty<c<0<d<\infty$. We show that $\alpha_{-}=c, \alpha_{+}=d$. Suppose that $\alpha_{+}<d$. Then there exists a sequence $\left\{\alpha_{n}\right\}_{n=1}^{\infty}$ such that $\alpha_{n} \rightarrow \alpha_{+}(n \rightarrow \infty)$ and $\Psi_{0}\left(\alpha_{n}\right) \in \mathcal{H}_{0}$. Suppose that $\Psi_{0}\left(\alpha_{+}\right) \in \mathcal{H}_{1}$. Then $\left(\Psi_{0}\left(\alpha_{n}\right), \Psi_{0}\left(\alpha_{+}\right)\right)=0$. Applying Lemma 2.4 to the case $\alpha=\alpha_{+}$, we have $\Psi_{0}\left(\alpha_{+}\right)=0$. But this is a contradiction. Hence $\Psi_{0}\left(\alpha_{+}\right) \in \mathcal{H}_{0}$. Then, in the same way as above, we can show that there exists a constant $\alpha^{\prime} \in\left(\alpha_{+}, d\right)$ such that $\Psi_{0}\left(\alpha^{\prime}\right) \in \mathcal{H}_{0}$. Hence, by the definition of $\alpha_{+}, \alpha^{\prime} \leq \alpha_{+}$. But this is a contradiction. Thus $\alpha_{+}=d$. Similarly we can show that $\alpha_{-}=c$. The same method works in the other cases of $\mathcal{I}$.

The proof of Theorem 2.1 shows in an obvious way that Theorem 2.1 can be generalized to the case of other eigenvectors of $H(\alpha)$ :

Theorem 2.5 Assume (A.1) and that $\mathcal{H}$ has the orthogonal decomposition (1.3) such that, for all $\alpha \in \mathcal{I}, H(\alpha)$ is reduced by $\mathcal{H}_{0}$. Suppose that, for each $\alpha \in \mathcal{I}, H(\alpha)$ has an isolated eigenvalue $E(\alpha)$ such that $\operatorname{dim} \operatorname{ker}(H(\alpha)-E(\alpha))=1, E(\cdot)$ is continuous on $\mathcal{I}$ and $\operatorname{ker}\left(H_{0}-E(0)\right) \subset \mathcal{H}_{0}$. Then, for all $\alpha \in \mathcal{I}$, $\operatorname{ker}(H(\alpha)-E(\alpha)) \subset \mathcal{H}_{0}$.

\subsection{Uniqueness of ground states}

We first prove a general fact on the stability of uniqueness of eigenvectors of $H(\alpha)$. 
Proposition 2.6 Assume (A.1). Suppose that, for each $\alpha \in \mathcal{I}$, there exist constants $E(\alpha) \in \mathbf{R}, \delta_{\alpha}>0$ and $K_{\alpha}>0$ such that

$$
\left[E(\alpha)-\delta_{\alpha}, E(\alpha)+\delta_{\alpha}\right] \cap \sigma(H(\alpha))=\{E(\alpha)\}
$$

and, for all $|\kappa|<K_{\alpha}$,

$$
\left[E(\alpha)-\delta_{\alpha}, E(\alpha)+\delta_{\alpha}\right] \cap \sigma(H(\alpha+\kappa))=\{E(\alpha+\kappa)\},
$$

so that $E(\alpha)$ is an eigenvalue of $H(\alpha)$. Suppose that $\operatorname{dim} \operatorname{ker}\left(H_{0}-E(0)\right)=1$. Then, for all $\alpha \in \mathcal{I}, \operatorname{dim} \operatorname{ker}(H(\alpha)-E(\alpha))=1$.

Proof. Let $a_{0}:=E(0)-\delta_{0}, b:=E(0)+\delta_{0}$. As in the proof of Lemma 2.4, we see that, for all $|\alpha|<\delta$ with some $\delta>0$ sufficiently small, $\operatorname{dim} \operatorname{Ran} P_{\alpha}\left(\left(a_{0}, b_{0}\right)\right)=\operatorname{dim} \operatorname{Ran} P_{0}\left(\left(a_{0}, b_{0}\right)\right)=$ 1. By $(2.7), \operatorname{Ran} P_{\alpha}\left(\left(a_{0}, b_{0}\right)\right)=\operatorname{ker}(H(\alpha)-E(\alpha)),|\alpha|<\delta$. Hence $\operatorname{dim} \operatorname{ker}(H(\alpha)-E(\alpha))=$ $1,|\alpha|<\delta$. Let

$$
\begin{aligned}
& a_{-}:=\inf \{\alpha \in \mathcal{I} \mid \operatorname{dim} \operatorname{ker}(H(\alpha)-E(\alpha))=1\} \\
& a_{+}:=\sup \{\alpha \in \mathcal{I} \mid \operatorname{dim} \operatorname{ker}(H(\alpha)-E(\alpha))=1\} .
\end{aligned}
$$

By the above fact, we have $a_{-}<0<a_{+}$. Consider the case $\mathcal{I}=(c, d)$ with $-\infty<c<$ $0<d<\infty$. We show that $a_{-}=c, a_{+}=d$. Suppose that $a_{+}<d$. Then there exists a sequence $\left\{\alpha_{n}\right\}_{n=1}^{\infty}$ such that $\alpha_{n} \rightarrow a_{+}(n \rightarrow \infty)$ and $\operatorname{dim} \operatorname{ker}\left(H\left(\alpha_{n}\right)-E\left(\alpha_{n}\right)\right)=1$. Suppose that $\operatorname{dim} \operatorname{ker}\left(H\left(a_{+}\right)-E\left(a_{+}\right)\right) \geq 2$. We have for all $n \geq n_{0}$ with some $n_{0} \geq 1$

$$
\operatorname{dim} \operatorname{Ran} P_{\alpha_{n}}\left(\left(E\left(a_{+}\right)-\delta_{a_{+}}, E\left(a_{+}\right)+\delta_{a_{+}}\right)\right)=\operatorname{dim} \operatorname{Ran} P_{a_{+}}\left(\left(E\left(a_{+}\right)-\delta_{a_{+}}, E\left(a_{+}\right)+\delta_{a_{+}}\right)\right) .
$$

Hence, for all $n \geq n_{0}, \operatorname{dim} \operatorname{Ran} P_{\alpha_{n}}\left(\left(E\left(a_{+}\right)-\delta_{a_{+}}, E\left(a_{+}\right)+\delta_{a_{+}}\right)\right) \geq 2$. By $(2.7)$,

$$
\operatorname{Ran} P_{\alpha_{n}}\left(\left(E\left(a_{+}\right)-\delta_{a_{+}}, E\left(a_{+}\right)+\delta_{a_{+}}\right)\right)=\operatorname{ker}\left(H\left(\alpha_{n}\right)-E\left(\alpha_{n}\right)\right), n \geq n_{0}
$$

which implies $\operatorname{dim} \operatorname{Ran} P_{\alpha_{n}}\left(\left(E\left(a_{+}\right)-\delta_{a_{+}}, E\left(a_{+}\right)+\delta_{a_{+}}\right)\right)=1$. But this is a contradiction. Thus $a_{+}=d$. Similarly we can show that $a_{-}=c$. The same method works in the other cases of $\mathcal{I}$.

We consider a sufficient condition for (2.6) and (2.7) to hold in the case $E(\alpha)=E_{0}(\alpha)$. Let

$$
E_{1}(\alpha):=\inf \left\{\sigma(H(\alpha)) \backslash\left\{E_{0}(\alpha)\right\}\right\}
$$

Proposition 2.7 Assume (A.1) and (A.2). Suppose that, for every $\alpha \in \mathcal{I}$, there exists a constant $L_{\alpha}>0$ such that

$$
\begin{gathered}
\alpha \pm L_{\alpha} \in \mathcal{I}, \\
\inf _{0 \leq|\kappa| \leq L_{\alpha}}\left\{E_{1}(\alpha+\kappa)-E_{0}(\alpha+\kappa)\right\}>E_{0}(\alpha)-\inf _{0 \leq|\kappa| \leq L_{\alpha}} E_{0}(\alpha+\kappa) .
\end{gathered}
$$

Then $H(\alpha)$ satisfies (2.6) and (2.7). 
Proof. Fix $\alpha \in \mathcal{I}$ arbitrarily. By (2.10), there is a real constant $M_{\alpha}$ such that

$$
\inf _{0 \leq|\kappa| \leq L_{\alpha}}\left\{E_{1}(\alpha+\kappa)-E_{0}(\alpha+\kappa)\right\}>M_{\alpha}>E_{0}(\alpha)-\inf _{0 \leq|\kappa| \leq L_{\alpha}} E_{0}(\alpha+\kappa) .
$$

Hence, for every $\kappa$ with $0 \leq|\kappa| \leq L_{\alpha}$, we have

$$
M_{\alpha}<E_{1}(\alpha+\kappa)-E_{0}(\alpha+\kappa) .
$$

In particular, putting $\kappa=0$, we have

$$
E_{0}(\alpha)+M_{\alpha}<E_{1}(\alpha) .
$$

By the second inequality in (2.11), there exists a constant $\delta_{\alpha}$ such that

$$
0<\delta_{\alpha}<M_{\alpha}+\inf _{0 \leq|\kappa| \leq L_{\alpha}} E_{0}(\alpha+\kappa)-E_{0}(\alpha)
$$

By (2.12) and (2.14), we have

$$
\begin{aligned}
E_{0}(\alpha)+\delta_{\alpha} & <M_{\alpha}+\inf _{0 \leq\left|\kappa^{\prime}\right| \leq L_{\alpha}} E_{0}\left(\alpha+\kappa^{\prime}\right) \\
& \leq\left(E_{1}(\alpha+\kappa)-E_{0}(\alpha+\kappa)\right)+E_{0}(\alpha+\kappa) \\
& =E_{1}(\alpha+\kappa)
\end{aligned}
$$

for $0 \leq|\kappa| \leq L_{\alpha}$, which, together with Lemma 2.3 and (2.9), implies (2.6) and (2.7).

Propositions 2.6 and 2.7 immediately yield the following theorem.

Theorem 2.8 Let the assumption of Proposition 2.7 be satisfied. Suppose that the ground state of $H_{0}$ is unique. Then, for all $\alpha \in \mathcal{I}$, the ground state of $H(\alpha)$ is unique.

A sufficient condition for (2.9) and (2.10) to hold is given in the following proposition.

Proposition 2.9 Assume (A.1) and (A.2). Suppose that $E_{0}(\alpha)<E_{1}(\alpha)$ for all $\alpha \in \mathcal{I}$, and $E_{1}(\alpha)$ is continuous in $\alpha \in \mathcal{I}$. Then (2.9) and (2.10) hold.

Proof. Fix $\alpha \in \mathcal{I}$ arbitrarily. Let $\varepsilon$ be such that

$$
0<\varepsilon<\frac{E_{1}(\alpha)-E_{0}(\alpha)}{3}
$$

By Lemma 2.3, there exists a constant $K_{0, \alpha}>0$ such that if $0 \leq|\kappa| \leq K_{0, \alpha}$, then $\alpha \pm K_{0, \alpha} \in \mathcal{I}$ and

$$
\left|E_{0}(\alpha)-E_{0}(\alpha+\kappa)\right|<\varepsilon
$$


Since $E_{1}(\alpha)$ is continuous in $\alpha \in \mathcal{I}$ by the present assumption, there exists a constant $K_{1, \alpha}>0$ such that if $0 \leq|\kappa| \leq K_{1, \alpha}$, then $\alpha \pm K_{1, \alpha} \in \mathcal{I}$ and

$$
\left|E_{1}(\alpha)-E_{1}(\alpha+\kappa)\right|<\varepsilon .
$$

Let

$$
L_{\alpha}:=\min \left\{K_{0, \alpha}, K_{1, \alpha}\right\} .
$$

Then $\alpha \pm L_{\alpha} \in \mathcal{I}$, i.e., (2.9) holds. By Lemma 2.3, there exists a constant $\kappa_{0}$ with $0 \leq\left|\kappa_{0}\right| \leq L_{\alpha}$ such that

$$
\inf _{0 \leq|\kappa| \leq L_{\alpha}} E_{0}(\alpha+\kappa)=E_{0}\left(\alpha+\kappa_{0}\right) .
$$

Hence we have

$$
\left|E_{0}(\alpha)-\inf _{0 \leq|\kappa| \leq L_{\alpha}} E_{0}(\alpha+\kappa)\right|=\left|E_{0}(\alpha)-E_{0}\left(\alpha+\kappa_{0}\right)\right|<\varepsilon .
$$

Since $E_{1}(\alpha)-E_{0}(\alpha)$ is continuous in $\alpha \in \mathcal{I}$, there exists a constant $\kappa_{1}$ with $0 \leq\left|\kappa_{1}\right| \leq L_{\alpha}$ such that

$$
\inf _{0 \leq|\kappa| \leq L_{\alpha}}\left\{E_{1}(\alpha+\kappa)-E_{0}(\alpha+\kappa)\right\}=E_{1}\left(\alpha+\kappa_{1}\right)-E_{0}\left(\alpha+\kappa_{1}\right),
$$

Hence we have by (2.15), (2.16), (2.17) and (2.19)

$$
\begin{aligned}
& \inf _{0 \leq|\kappa| \leq L_{\alpha}}\left\{E_{1}(\alpha+\kappa)-E_{0}(\alpha+\kappa)\right\} \\
= & E_{1}\left(\alpha+\kappa_{1}\right)-E_{0}\left(\alpha+\kappa_{1}\right) \\
= & \left(E_{1}\left(\alpha+\kappa_{1}\right)-E_{1}(\alpha)\right)+\left(E_{0}(\alpha)-E_{0}\left(\alpha+\kappa_{1}\right)\right)+\left(E_{1}(\alpha)-E_{0}(\alpha)\right) \\
\geq & -2 \varepsilon+\left(E_{1}(\alpha)-E_{0}(\alpha)\right) \\
> & \varepsilon \\
> & \left|E_{0}(\alpha)-\inf _{0 \leq|\kappa| \leq L_{\alpha}} E_{0}(\alpha+\kappa)\right|
\end{aligned}
$$

Thus (2.10) follows.

Theorem 2.8 and Proposition 2.9 imply the following theorem:

Theorem 2.10 Assume (A.1), (A.2) and that $E_{0}(\alpha)<E_{1}(\alpha)$ for all $\alpha \in \mathcal{I}$ and $E_{1}(\alpha)$ is continuous in $\alpha \in \mathcal{I}$. Suppose that the ground state of $H_{0}$ is unique. Then, for all $\alpha \in \mathcal{I}$, the ground state of $H(\alpha)$ is unique. 


\section{A Special Class of Self-adjoint Operators}

Let $H_{I}$ be a symmetric operator on $\mathcal{H}$ satisfying the following condition:

(B.1) $D\left(H_{0}\right) \subset D\left(H_{I}\right)$ and there exist constants $a, b>0$ such that, for all $\psi \in D\left(H_{0}\right)$,

$$
\left\|H_{I} \psi\right\| \leq a\left\|H_{0} \psi\right\|+b\|\psi\|
$$

We define

$$
T(\alpha):=H_{0}+\alpha H_{I}
$$

with $\alpha \in \mathbf{R}$ a coupling constant. Let $\mathcal{I}_{a}$ be an open interval from $-1 / a$ to $1 / a$ :

$$
\mathcal{I}_{a}:=\left(-\frac{1}{a}, \frac{1}{a}\right)
$$

By the Kato-Rellich theorem (e.g., [RS2, Theorem X.12]), for all $\alpha \in \mathcal{I}_{a}, T(\alpha)$ is selfadjoint with $D(T(\alpha))=D\left(H_{0}\right)$ and bounded from below with

$$
E_{0}(T(\alpha)) \geq E_{0}-\max \left\{\frac{b|\alpha|}{1-a|\alpha|},|\alpha|\left(a\left|E_{0}\right|+b\right)\right\}
$$

where

$$
E_{0}:=E_{0}\left(H_{0}\right)
$$

We assume the following:

(B.2) For all $\alpha \in \mathcal{I}_{a}, E_{0}(T(\alpha))$ is an isolated eigenvalue of $T(\alpha)$.

Theorem 3.1 Assume (B.1), (B.2) and that $\mathcal{H}$ has the orthogonal decomposition (1.3) such that, for all $\alpha \in \mathcal{I}_{a}, T(\alpha)$ is reduced by $\mathcal{H}_{0}$. Suppose that, for all $\alpha \in \mathcal{I}_{a}$, the ground state $T(\alpha)$ is unique and that the ground state of $H_{0}$ is in $\mathcal{H}_{0}$. Then, for all $\alpha \in \mathcal{I}_{a}$, the ground state of $T(\alpha)$ is in $\mathcal{H}_{0}$.

Corollary 3.2 Assume (B.1), (B.2) and that $\mathcal{H}$ has the orthogonal decomposition (1.3) such that, for all $\alpha \in \mathcal{I}_{a}, T(\alpha)$ is reduced by $\mathcal{H}_{0}$. Suppose that the ground state of $H_{0}$ is unique and in $\mathcal{H}_{0}$. Moreover, suppose that there exists an $\alpha^{\prime} \in \mathcal{I}_{a}$ such that $T\left(\alpha^{\prime}\right)$ has a ground state which is not in $\mathcal{H}_{0}$. Then, for some $\alpha_{0} \in \mathcal{I}_{a} \backslash\{0\}$, the ground state of $T\left(\alpha_{0}\right)$ is degenerate.

We prove these results by applying Theorem 2.1 and Corollary 2.2. To do this we need a lemma.

Let

$$
Q_{z}(\alpha):=(T(\alpha)-z)^{-1}, \quad z \in \rho(T(\alpha))
$$


Lemma 3.3 Assume (B.1). Then, for all $z \in \mathbf{C} \backslash \mathbf{R}$, the operator-valued function: $\alpha \rightarrow Q_{z}(\alpha)$ is continuous on $\mathcal{I}_{a}$ in operator norm topology.

Proof. Fix $\alpha \in \mathcal{I}_{a}$ and $z \in \mathbf{C} \backslash \mathbf{R}$ arbitrarily. Since $D(T(\alpha))=D(T(\alpha+\kappa))=D\left(H_{0}\right)$ for every $\kappa \in \mathbf{R}$ with $\alpha+\kappa \in \mathcal{I}$, we have

$$
Q_{z}(\alpha+\kappa)-Q_{z}(\alpha)=-\kappa Q_{z}(\alpha+\kappa) H_{I} Q_{z}(\alpha)
$$

For $\Psi \in D\left(H_{0}\right)$, we have by the triangle inequality and (3.1)

$$
\begin{aligned}
\left\|H_{0} \Psi\right\| & \leq\|T(\alpha) \Psi\|+|\alpha|\left\|H_{I} \Psi\right\| \\
& \leq\|T(\alpha) \Psi\|+a|\alpha|\left\|H_{0} \Psi\right\|+b|\alpha|\|\Psi\| .
\end{aligned}
$$

Hence

$$
\left\|H_{0} \Psi\right\| \leq \frac{1}{1-a|\alpha|}\|T(\alpha) \Psi\|+\frac{b|\alpha|}{1-a|\alpha|}\|\Psi\|
$$

where $|\alpha|$ satisfies that $0<|\alpha|<1 / a$. Putting this into (3.1), we obtain

$$
\left\|H_{I} \Psi\right\| \leq \frac{a}{1-|\alpha| a}\|T(\alpha) \Psi\|+\left(\frac{a b|\alpha|}{1-a|\alpha|}+b\right)\|\Psi\|,
$$

which implies that $H_{I} Q_{z}(\alpha)$ is bounded. Since $\left\|Q_{z}(\alpha+\kappa)\right\| \leq 1 /|\Im z|$, we obtain

$$
\left\|Q_{z}(\alpha+\kappa)-Q_{z}(\alpha)\right\| \leq \frac{|\kappa|}{|\Im z|}\left\|H_{I} Q_{z}(\alpha)\right\| \rightarrow 0
$$

as $\kappa \rightarrow 0$. Hence the desired result follows.

\section{Proof of Theorem 3.1}

By th present assumption, (3.4) and Lemma 3.3, the assumption of Theorem 2.1 with $H(\alpha)=T(\alpha)$ and $\mathcal{I}=\mathcal{I}_{a}$ is satisfied. Thus the assertion follows.

Remark 3.1 Assume (B.1) and fix $\alpha \in \mathcal{I}_{a}$ arbitrarily. Then $T(\alpha+\kappa)$ is an analytic family of type (A) near $\kappa=0$. This follows from (3.8) and a general fact [RS4, p.16, Lemma].

We can obtain results on uniqueness of ground states of $T(\alpha)$ by applying the results in $\S 2.2$ to the operator $T(\alpha)$. But we omit writing down them. 


\section{Application to the WW Model}

In this section we apply the main results of Section 3 to the WW model. We first recall the definition of the WW model.

We take a Hilbert space of bosons to be

$$
\mathcal{F}_{\mathrm{b}}:=\mathcal{F}_{\mathrm{b}}\left(L^{2}\left(\mathbf{R}^{d}\right)\right):=\bigoplus_{n=0}^{\infty}\left[\otimes_{\mathrm{sym}}^{n} L^{2}\left(\mathbf{R}^{d}\right)\right]
$$

$(d \in \mathbf{N})$ the symmetric Fock space over $L^{2}\left(\mathbf{R}^{d}\right)\left(\otimes_{\mathrm{s}}^{n} \mathcal{K}\right.$ denotes the $n$-fold symmetric tensor product of a Hilbert space $\mathcal{K}, \otimes_{\mathrm{s}}^{0} \mathcal{K}:=\mathbf{C}$ ). In this paper, we set both of $\hbar$ (the Planck constant divided by $2 \pi$ ) and $c$ (the speed of light) one, i.e., $\hbar=c=1$.

Let $\omega: \mathbf{R}^{d} \rightarrow[0, \infty)$ be Borel measurable such that $0<\omega(k)<\infty$ for almost everywhere (a.e.) $k \in \mathbf{R}^{d}$ with respect to the $d$-dimensional Lebesgue measure and

$$
H_{\mathrm{b}}:=d \Gamma(\omega),
$$

the second quantization of the multiplication operator on $L^{2}\left(\mathbf{R}^{d}\right)$ by the function $\omega[\mathrm{RS} 2$, $\S \mathrm{X} .7]$.

Let $\lambda$ be a function on $\mathbf{R}^{d}$. We assume the following (W.1) and (W.2):

(W.1) The function $\lambda$ is continuous on $\mathbf{R}^{d}$, not identically zero with $\lambda, \lambda / \omega \in L^{2}\left(\mathbf{R}^{d}\right)$.

(W.2) The function $\omega(k)$ is continuous with

$$
\lim _{|k| \rightarrow \infty} \omega(k)=\infty
$$

and there exist constants $\gamma_{\omega}>0$ and $C_{\omega}>0$ such that

$$
\left|\omega(k)-\omega\left(k^{\prime}\right)\right| \leq C_{\omega}\left|k-k^{\prime}\right|^{\gamma_{\omega}}\left(1+\omega(k)+\omega\left(k^{\prime}\right)\right), \quad k, k^{\prime} \in \mathbf{R}^{d} .
$$

We define a matrix $c$ by

$$
c:=\left(\begin{array}{cc}
0 & 0 \\
1 & 0
\end{array}\right)
$$

The Hamiltonian $H_{\mathrm{ww}}(\alpha)$ of the WW model is defined by

$$
H_{\mathrm{ww}}(\alpha):=H_{0}+\alpha H_{I}
$$

acting in

$$
\mathcal{H}=\mathbf{C}^{2} \otimes \mathcal{F}_{\mathrm{b}}
$$


with

$$
\begin{aligned}
& H_{0}:=\mu_{0} c^{*} c \otimes I+I \otimes H_{\mathrm{b}}, \\
& H_{I}:=c^{*} \otimes a(\lambda)+c \otimes a(\lambda)^{*},
\end{aligned}
$$

where $\mu_{0}, \alpha \in \mathbf{R} \backslash\{0\}$ are constant parameters and $a(\cdot)$ (resp. I) denotes the annihilation operator on $\mathcal{F}_{\mathrm{b}}$ (resp. identity operator). It is easy to prove the following fact:

Lemma 4.1 (i) The operator $H_{I}$ is infinitesimally small with respect to $H_{0}$.

(ii) For all $\alpha \in \mathbf{R}, H_{\mathrm{ww}}(\alpha)$ is self-adjoint with $D\left(H_{\mathrm{ww}}(\alpha)\right)=D\left(H_{0}\right)$ and bounded from below.

The WW model has a conservation law for a kind of the particle number in the sense described below. Let $\sigma_{3}$ be the third of the Pauli matrices:

$$
\sigma_{3}:=\left(\begin{array}{cc}
1 & 0 \\
0 & -1
\end{array}\right)
$$

and define

$$
N_{P}:=\frac{1+\sigma_{3}}{2} \otimes I+I \otimes N_{\mathrm{b}}
$$

where $N_{\mathrm{b}}:=d \Gamma(I)$ is the boson number operator. The operator $N_{P}$ was introduced in $\left[\right.$ HS95, §6]. Let $P^{(\ell)}$ be the orthogonal projection onto the $\ell$-particle space of $\mathcal{F}_{\mathrm{b}}(\ell \geq 0)$. Then we have

$$
N_{\mathrm{b}}=\sum_{\ell=0} \ell P^{(\ell)}
$$

The spectral resolution of $N_{P}$ is given by

$$
N_{P}=\sum_{\ell=0} \ell P_{\ell}
$$

where

$$
P_{\ell}:= \begin{cases}\frac{1-\sigma_{3}}{2} \otimes P^{(0)} & \text { if } \ell=0, \\ \frac{1+\sigma_{3}}{2} \otimes P^{(\ell-1)}+\frac{1-\sigma_{3}}{2} \otimes P^{(\ell)} & \text { if } \ell \in \mathbf{N} .\end{cases}
$$

It is easy to see that, for every $\alpha \in \mathbf{R}$ and each $\ell \in\{0\} \cup \mathbf{N}$,

$$
P_{\ell} H_{\mathrm{ww}}(\alpha) \subset H_{\mathrm{ww}}(\alpha) P_{\ell} .
$$

Hence $H_{\mathrm{ww}}(\alpha)$ is reduced by $P_{\ell} \mathcal{H}$. 
Let

$$
\mathcal{H}_{0}:=\left(P_{0}+P_{1}\right) \mathcal{H}
$$

and

$$
\left.\mathcal{H}_{1}:=\mathcal{H}_{0}^{\perp} \text { (the orthogonal complement of } \mathcal{H}_{0}\right)
$$

Then

$$
\mathcal{H}=\mathcal{H}_{0} \oplus \mathcal{H}_{1}
$$

The following lemma easily follows:

Lemma 4.2 (i) For each $\alpha \in \mathbf{R}, H_{\mathrm{ww}}(\alpha)$ is reduced by $\mathcal{H}_{j}, j=1,2$.

(ii) $H_{0}$ has a unique ground state in $\mathcal{H}_{0}$.

Let

$$
E_{0}^{\mathrm{Ww}}(\alpha):=E_{0}\left(H_{\mathrm{wW}}(\alpha)\right)
$$

and

$$
\mu:=\text { ess. } \inf _{k \in \mathbf{R}^{d}} \omega(k) \geq 0 .
$$

We say that the WW model is massive (resp. massless) if $\mu>0$ (resp. $\mu=0)$.

Proposition 4.3 ([Ar99, Remark 3.1], [AH99, Proposition 6.10(i)])

$$
\sigma\left(H_{\mathrm{ww}}(\alpha)\right)=\left[E_{0}^{\mathrm{ww}}(\alpha)+\mu, \infty\right),
$$

where $\sigma_{\text {ess }}(\cdot)$ denotes essential spectrum.

We define

$$
D_{\mu}^{\alpha}(z):=-z+\mu_{0}-\alpha^{2} \int_{\mathbf{R}^{d}} d k \frac{|\lambda(k)|^{2}}{\omega(k)-z}, \quad z \in \mathbf{C}_{\mu}:=\mathbf{C} \backslash[\mu, \infty)
$$

The limit

$$
C_{\mu}:=\lim _{t \downarrow 0} \int_{\mathbf{R}^{d}} d k \frac{|\lambda(k)|^{2}}{\omega(k)-\mu+t}
$$

exists or is infinity. In the former case, $C_{\mu}>0$ by (W.1). It is easy to see that $D_{\mu}^{\alpha}(x)$ is monotone decreasing in $x<\mu$. Hence the limit

$$
d_{\mu}^{\alpha}:=\lim _{x \uparrow \mu} D_{\mu}^{\alpha}(x)
$$

exists or is $-\infty$ and

$$
d_{\mu}^{\alpha}=-\mu+\mu_{0}-\alpha^{2} C_{\mu} .
$$


Let

$$
\beta_{0}:= \begin{cases}\frac{\mu_{0}-\mu}{C_{\mu}} & \text { if } 0<C_{\mu}<\infty \\ 0 & \text { if } C_{\mu}=\infty\end{cases}
$$

and

$$
\mathcal{A}_{\mu}:=\left\{\alpha \in \mathbf{R} \mid-\infty \leq d_{\mu}^{\alpha}<0\right\}=\left\{\alpha \in \mathbf{R} \mid \alpha^{2}>\beta_{0}\right\} .
$$

For all $\alpha \in \mathcal{A}_{\mu}$, there exists a unique zero $E_{\mathrm{ww}}(\alpha)$ of $D_{\mu}^{\alpha}(z)$ :

$$
E_{\mathrm{wW}}(\alpha)=\mu_{0}-\alpha^{2} \int_{\mathbf{R}^{d}} d k \frac{|\lambda(k)|^{2}}{\omega(k)-E_{\mathrm{wW}}(\alpha)} .
$$

Proposition 4.4 ([H99, Theorem 2.3 (b),(c)]) Let $\alpha \in \mathcal{A}_{\mu}$. Assume either (i) $\mu>0$ or (ii) $\mu=0$ with $\nabla \omega \in L^{\infty}\left(\mathbf{R}^{d}\right)$. Then there exists a constant $\alpha_{\mathrm{ww}} \in \mathcal{A}_{\mu} \cap(0, \infty)$ such that, for all $|\alpha|>\alpha_{\mathrm{ww}}$,

$$
\left\{E_{0}^{\mathrm{ww}}(\alpha), E_{\mathrm{ww}}(\alpha), 0\right\} \subset \sigma_{\mathrm{p}}\left(H_{\mathrm{ww}}(\alpha)\right)
$$

with

$$
E_{0}^{\mathrm{Ww}}(\alpha)<\min \left\{E_{\mathrm{ww}}(\alpha), 0\right\}
$$

and

$$
\Psi_{0}(\alpha) \notin \mathcal{H}_{0}
$$

Let

$$
E_{1}^{\mathrm{ww}}(\alpha):=\inf \left\{\sigma\left(H_{\mathrm{ww}}(\alpha)\right) \backslash\left\{E_{0}^{\mathrm{ww}}(\alpha)\right\}\right\}
$$

and

$$
\varepsilon_{0}:=\min \left\{0, \mu_{0}\right\}, \quad \varepsilon_{1}:=\max \left\{0, \mu_{0}\right\} .
$$

Note that, if $E_{1}^{\mathrm{ww}}(\alpha)$ is an eigenvalue of $H_{\mathrm{ww}}(\alpha)$, then each eigenvector corresponding to it physically describes one of the first excited states of the WW model.

Theorem 4.5 Let $\mu>0$. Then:

(i) There exists a constant $\alpha_{0} \in \mathcal{A}_{\mu}$ such that $H_{\mathrm{ww}}\left(\alpha_{0}\right)$ has degenerate ground states.

(ii) There exists a constant $\alpha_{1} \in \mathcal{A}_{\mu}$ such that $E_{1}^{\mathrm{ww}}\left(\alpha_{1}\right)$ is an eigenvalue of $H_{\mathrm{ww}}\left(\alpha_{1}\right)$ and

$$
E_{1}^{\mathrm{Ww}}\left(\alpha_{1}\right)<E_{0}^{\mathrm{Ww}}\left(\alpha_{1}\right)+\mu=\inf \sigma\left(H_{\mathrm{ww}}\left(\alpha_{1}\right)\right) .
$$

Moreover, if $0<\mu<\left|\mu_{0}\right|$, then

$$
E_{1}^{\mathrm{WW}}\left(\alpha_{1}\right)<\varepsilon_{1}
$$


Proof. (i) Since $\mu>0$, it follows from [AH97, Theorem 1.2] that, for all $\alpha \in \mathbf{R}$, $H_{\mathrm{ww}}(\alpha)$ has a ground state and $E_{0}^{\mathrm{ww}}(\alpha)$ is an isolated eigenvalue of $H_{\mathrm{ww}}(\alpha)$. These facts together with Lemmas 4.1, 4.2, Proposition 4.4 imply that the assumption of Corollary 3.2 with $T(\alpha)=H_{\mathrm{ww}}(\alpha)$ is satisfied. Hence there exists a constant $\alpha_{0} \neq 0$ such that the ground state of $H_{\mathrm{ww}}\left(\alpha_{0}\right)$ is degenerate. If $\alpha_{0} \notin \mathcal{A}_{\mu}$ so that $d_{\mu}^{\alpha_{0}} \geq 0$, then, by [AH99, Theorem 6.14(i)], $H_{\mathrm{ww}}\left(\alpha_{0}\right)$ has a unique ground state. But this is a contradiction.

(ii) By Lemma 4.3, we have for all $\alpha \in \mathbf{R}$

$$
E_{0}^{\mathrm{WW}}(\alpha)<E_{1}^{\mathrm{Ww}}(\alpha) \leq E_{0}^{\mathrm{Ww}}(\alpha)+\mu .
$$

Suppose that, for all $\alpha \in \mathbf{R} \backslash\{0\}$,

$$
E_{1}^{\mathrm{wW}}(\alpha)=\inf \sigma_{\mathrm{ess}}\left(H_{\mathrm{wW}}(\alpha)\right)=E_{0}^{\mathrm{ww}}(\alpha)+\mu .
$$

By an application of Lemma 2.4, $E_{0}^{\mathrm{ww}}(\alpha)$ is continuous in $\alpha \in \mathbf{R}$. Hence so is $E_{1}^{\mathrm{ww}}(\alpha)$. Then, by an application of Theorem 2.10, for all $\alpha \in \mathbf{R}$, the ground state of $H_{\mathrm{ww}}(\alpha)$ is unique. But this contradicts part (i). Hence there exists a constant $\alpha_{1} \neq 0$ such that (4.28) holds and $E_{1}^{\mathrm{ww}}\left(\alpha_{1}\right)$ is an eigenvalue of $H_{\mathrm{ww}}\left(\alpha_{1}\right)$. We show that $\alpha_{1} \in \mathcal{A}_{\mu}$. If $\mu_{0}<0$, then $d_{\mu}^{\alpha}<0$ for all $\alpha \in \mathbf{R}$, which implies $\mathcal{A}_{\mu}^{\alpha}=\mathbf{R}\left(\mu_{0}<0\right)$. Hence $\alpha_{1} \in \mathcal{A}_{\mu}$. Let $\mu_{0}>0$. Suppose that $d_{\mu}^{\alpha_{1}} \geq 0$. Then, by [AH99, Theorem 6.14(i)] we have $E_{1}^{\mathrm{ww}}\left(\alpha_{1}\right)=$ $E_{0}^{\mathrm{Ww}}\left(\alpha_{1}\right)+\mu$, which contradicts (4.28). Hence $d_{\mu}^{\alpha_{1}}<0$. Therefore $\alpha_{1} \in \mathcal{A}_{\mu}$.

Finally we prove (4.29). Let $\mu<\left|\mu_{0}\right|$. Since $0 \in \sigma_{\mathrm{p}}\left(H_{\mathrm{ww}}(\alpha)\right)$ for all $\alpha \in \mathbf{R}$ by [AH99, Proposition 6.13], we have

$$
E_{1}^{\mathrm{WW}}\left(\alpha_{1}\right)<E_{0}^{\mathrm{WW}}\left(\alpha_{1}\right)+\mu \leq 0+\mu=\mu .
$$

We first consider the case $0<\mu_{0}$. In this case, $\varepsilon_{0}=0, \varepsilon_{1}=\mu_{0}$. Hence $E_{1}^{\mathrm{ww}}\left(\alpha_{1}\right)<\varepsilon_{1}$. We next consider the case $\mu_{0}<0$. In this case, $\varepsilon_{0}=\mu_{0}$ and $\varepsilon_{1}=0$. Since $\alpha_{1} \in \mathcal{A}_{\mu}$ (i.e., $\left.d_{\mu}^{\alpha_{1}}<0\right)$, we have by [AH99, Proposition 6.13 (ii)] $0, E_{\mathrm{ww}}\left(\alpha_{1}\right) \in \sigma_{\mathrm{p}}\left(H_{\mathrm{ww}}(\alpha)\right)$ with $E_{\mathrm{ww}}\left(\alpha_{1}\right)<0$. Since $\mu_{0}<0$, we have

$$
D_{\mu}^{\alpha_{1}}\left(\mu_{0}\right)=-\alpha_{1}^{2} \int_{\mathbf{R}^{d}} d k \frac{|\lambda(k)|^{2}}{\omega(k)-\mu_{0}}<0 .
$$

This implies that $E_{\mathrm{ww}}\left(\alpha_{1}\right)<\mu_{0}$, since $D_{\mu}^{\alpha_{1}}(x)$ is monotone decreasing in $x<\mu$ and $D_{\mu}^{\alpha_{1}}\left(E_{\mathrm{ww}}\left(\alpha_{1}\right)\right)=0$. Hence we have

$$
E_{1}^{\mathrm{WW}}\left(\alpha_{1}\right)<E_{0}^{\mathrm{WW}}\left(\alpha_{1}\right)+\mu \leq E_{\mathrm{wW}}\left(\alpha_{1}\right)+\mu<\mu_{0}+\mu<0=\varepsilon_{1} .
$$

Thus (4.29) follows.

Remark 4.1 Generally speaking, in a quantum field model, it is difficult to prove the existence of an eigenvalue corresponding to the first excited states of the model. There are many papers stating the possibility of the existence of the first excited states, but, to 
authors' best knowledge, there is few papers pointing out the real existence of those. In this sense, Theorem 4.5-(ii) has a meaning. Moreover, note that, if $0<\mu<\left|\mu_{0}\right|=\varepsilon_{1}-\varepsilon_{0}$, then $\varepsilon_{1}$ is an embedded eigenvalue of $H_{0}$. In this case too, Theorem 4.5-(ii) holds, showing that, in the WW model, the embedded eigenvalue does not necessarily disappear under the perturbation $\alpha H_{I}$. The phenomena mentioned in Theorem 4.5 do not occur in the region of the coupling constant treated by Hübner and Spohn [HS95, §6] and ourselves in [AH99, Theorem 6.14(i)].

Remark 4.2 We may expect that, in the massless case too (i.e. $\mu=0$ ), Theorem 4.5-(i) holds.

\section{Acknowledgments}

One (M. H.) of the authors would like to thank H. Spohn, F. Hiroshima, R. A. Minlos, H. Ezawa and K. Watanabe for their valuable advices. Research of M. H. is supported by the Grant-In-Aid No.11740109 for Encouragement of Young Scientists from Japan Society for the Promotion of Science (JSPS). A.A. is supported by the Grant-in-Aid No.11440036 for Scientific Research from the Ministry of Education, Science, Sports and Culture.

\section{References}

[Ar99] Arai, A.: Essential Spectrum of a Self-Adjoint Operators on an Abstract Hilbert Space of Fock Type and Applications to Quantum Field Hamiltonians, Hokkaido University Preprint Series in Mathematics \# 445, February 1999.

[AH97] Arai, A. and Hirokawa, M.: On the Existence and Uniqueness of Ground States of a Generalized Spin-Boson Model, J. Funct. Anal. 151 (1997), 455-503.

[AH99] Arai, A. and Hirokawa, M.: Ground States of a General Class of Quantum Field Hamiltonians, to appear in Rev. Math. Phys.. (mp_arc 99-179)

[H99] Hirokawa, M.: Remarks on the Ground State Energy of the Spin-Boson Model. An Application of the Wigner-Weisskopf Model, (preprint, 1999).

[HS95] Hübner, M. and Spohn, H.: Spectral properties of the spin-boson Hamiltonian, Ann. Inst. Henri. Poincaré 62 (1995), 289-323.

[Ka] Kato, T.: Perturbation Theory for Linear Operators, Springer-Verlag, Berlin Heidelberg New York, 1980. 
[RS1] Reed, M. and Simon, B.: Methods of Modern Mathematical Physics Vol. I, Academic Press, New York, 1975.

[RS2] Reed, M. and Simon, B.: Methods of Modern Mathematical Physics Vol. II, Academic Press, New York, 1975.

[RS4] Reed, M. and Simon, B.: Methods of Modern Mathematical Physics Vol. IV, Academic Press, New York, 1978.

[WW] Weisskopf, V. F. and Wigner, E. P.: Berechnung der natürlichen Linienbreite auf Grund der Diracschen Lichttheorie, Z. Phys. 63 (1930), 54-73. 